\title{
Soft Assistive Robot for personal care of elderly people
}

\author{
M. Manti, Student Member, IEEE, A. Pratesi, E. Falotico, Member, IEEE, M. Cianchetti, Member, \\ IEEE, C. Laschi, Senior Member, IEEE
}

\begin{abstract}
The authors focus on the possibility to adapt technologies and basic concepts of Soft Robotics for building a new generation of soft modular manipulator for assistive robotics that can safely come into direct contact with humans in a challenging scenario which is the bathing activity. This paper starts with the presentation of the concept of the modular manipulator and then moves toward a detailed description of one of its modules. The idea is to develop a manipulator which counts on an actuation system based on McKibben-based flexible fluidic actuators combined with motor driven cables, by addressing technological issues related to effectiveness and reliability. Shortening, elongation and bending capabilities have been assessed by testing different patterns of activations. These measures allowed the estimation of the single module performances and its workspace. These outcomes represent the starting point for the development of a novel modular manipulator to be used as a shower arm for bathing activities.
\end{abstract}

\section{INTRODUCTION}

Assistive robotics is employed for developing a set of technologies aiming to improve the independence and quality of life in everyday living [1-2]. Nowadays, the role played by this research area meets the needs given by the impact of demographic ageing, that represents a factor of major significance for the coming decades. This trend implies a growing number of older people living alone and in need of care due to age-related conditions and to loss of abilities [3]. When people are not able to perform the basic activities of daily living (ADLs), such as bathing, dressing, feeding, transferring, they become dependent on help from caregivers. As a result, difficulties in performing ADLs are an important predictor of (i) nursing care home use, (ii) use of hospital and healthcare services, (iii) use of physician services [4-6]. With the increasing achievements of technologies, robots are being developed in an user-oriented way with the purpose of assisting and interacting with people during everyday lives. They are mainly focused on ADLs by providing effective tools in order to improve the independent living and the quality of life [7]. One of the most critical and high risk ADL of the ageing population is

Research supported by the I-Support project in HORIZON 2020 PHC19, Grant Agreement \#643666 and the RoboSoft CA in FP7 ICT FET-Open project \#619319. The work is also supported by the Italian Ministry of Foreign Affairs and International Cooperation DGSP-UST through Joint Laboratory on Biorobotics Engineering project.

M.M., A.P., E.F., M.C. and C.L. are with the BioRobotics Institute of the Scuola Superiore Sant'Anna; e-mail: \{m.manti, a.pratesi, e.falotico, m.cianchetti, c.laschi@sssup.it\} the bathing activity [8]. A support in these activities, representing an early index of ADL disabilities, will improve independent living for persons prone to loss of autonomy, thus lightening the users' caring issues [1]. Although bathing is a basic ADL, only few approaches have tried to establish advanced assistive technology for this field so far. Literature analysis on assistive technologies for bathing task suggests only two commercially available shower devices: the Oasis Seated Shower system [9] and the Seat Lift Device [10]. The first one provides an automated assistance for bathing; it is bulky, expensive, completely lacking of active interaction with the user, thus the will of the user is not considered. The second one is simpler and partially automated; it helps positioning the user, by not providing any assistance during showering task. Hence, there is a need for an ICT-supported robotic system that will assist the elderly people in their hygiene tasks by compensating for their loss of ability. Among the main general requirements for a service robotics system that targets bathing tasks, there are safety, reliability, adaptability to users' actions [11-12]. Moreover, the system could be exploited in different real life applications, thus it should be modular and flexible for addressing a safe humanrobot interaction (HRI). In particular, this last issue is addressed and stressed in our technological concept because the assistive robot should come into direct contact and interact with the elderly people for accomplishing the task. Recent advancements on ICT and robotic technologies are providing the basis on which develop assistive robotic devices able of helping user independently accomplishing the task. The lack of technologies that satisfy the previous requirements paves the possibility of facing the challenge by exploiting soft robotics technologies. Our purpose is to apply, for the first time, soft robotics technologies in a new challenging scenario, like the showering activities, by involving a system which safely comes into direct contact with humans in an intimate task, providing a service and giving the sensitive nature of the bathing activity. In particular the aim is to design and develop a modular soft manipulator to be used as a shower arm.

\section{MATERIALS AND METHODS}

The purpose of the present work is to give an overview of a novel soft shower arm, designed and developed applying soft robotics principles, that would be able to help people bathing the worst reachable regions of their body.

\section{A. Manipulator overview}

Taking into account the workspace devoted to reach the target region of the users' body, we propose a modular system where one portion (the proximal one) is dedicated for positioning the arm by facing the gravity effects, the 
remaining part (made of two identical interconnected modules) is employed for accomplishing the typical bathing task (rinsing and scrubbing functionalities). In particular, the complete system will be made of three interconnected modules: the proximal one is only three cable-based; the middle and distal one are identical and embed three McKibben-based flexible fluidic actuators combined with three cables. In this preliminary phase, we focused on the choice of the suitable technologies to be involved in the design for addressing the following main issues: the need for the soft arm of covering the target workspace by exploiting shortening, elongation and bending; the need of a safe human-robot interaction; acceptability and adaptability to users' actions, mobility needs and capabilities. These requirements are coherent with the purpose of developing a modular and reusable/reconfigurable manipulator that results simple, reliable and dexterous. The final design comes out from two different aspects: (i) dimension of the complete modular shower arm in order to cover the target workspace; (ii) the type of actuation technology respect to the expected performances. A suitable trade-off between these features is the combination of cables and flexible fluidic actuators. From one side, the use of cables allows us to obtain multiple advantages, such as shortening, steering of the arm position, gravity effects compensation with a stiffness variation; at the same time, an exclusive cable-based actuation requires an higher number of modules for covering the same length of the other type. From the other one the elongation performances due to the fluidic actuators maximize the reachable length thus reducing the number of modules. Fig. 1 gives an overview of a possible scenario with two soft robotic arms (with identical dimensions and functionalities) mounted on the bathroom wall respect to the user that, seated on a chair, approaches the robotic shower. The distance between the wall and the longitudinal axes of the user is approximately $500 \mathrm{~mm}$. The position of the opposite shoulder respect to the wall (located at around $750 \mathrm{~mm}$ from the wall), delimits the workspace boundaries. The arm position is defined according to the regions of the users' body to reach, such as the back region and the lower limbs that are the most critical to approach for an user. The robotic shower comes out from the concept of having a system that helps user to perform independently the bathing task, by washing specific portions of the body not reachable by the user himself.

\section{B. Design and manufacturing of the module}

The starting point for delivering our technological concept of the robotic shower arm has been a literature analysis regarding the design of manipulators based on variable stiffness mechanisms. The challenge of developing manipulators that rely on fluidic actuators and cables has been largely faced in the surgical robotics field by opening the door to other applications [13]. Good examples of soft manipulators based on tendons pulled in relation to the chambers pressurized are: the KSI tentacle manipulator powered by a hybrid system of pneumatic bellows and electric motors [14]; the OCTARM continuum robot [15] that is able to perform adaptive manipulation in challenging

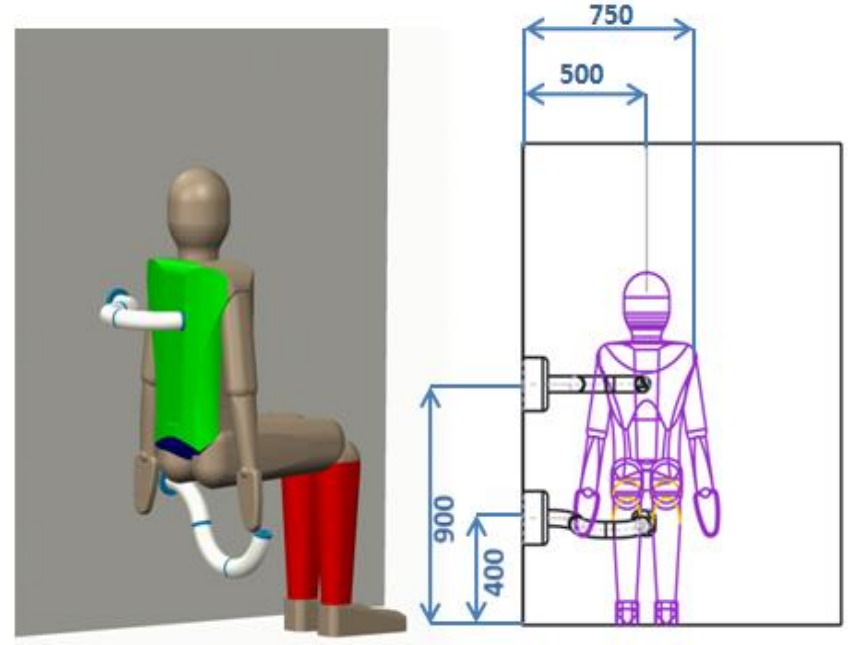

Figure 1. Concept overview: soft robotic shower arms mounted on the wall. The upper arm will be dedicated for the back region, the lower arm for the lower limbs.

environments by using air muscle actuators; the Air-Octor manipulator that uses three cables and a single chamber [16]; the continuum robot proposed by Pritts [17] uses 6-8 contracting/extending McKibben actuators for providing two-axis bending.

Taking inspiration from these works, our device is designed to rely on a combination of three cables, for shortening and bending movements, and three McKibben-based flexible fluidic actuators, for their potentiality of enabling bending and elongation movements in soft structures [18]. The difference of our McKibben-based actuators, respect to the traditional ones implemented in [19-21] regards a functional modification to the external braided structure usually placed outside the elastomeric chamber. Taking into account the basic elements which a traditional McKibben is made of, we selected the following components in order to realize our flexible fluidic actuator: (i) a balloon made of latex silicone rubber acting as internal chamber; (ii) two end-caps that delimit the actuator length (one is used to house the air source tube connector); (iii) an external braided structure with a bellows shape.

A braided sheath (Pro Power PETBK3B10) with a nominal internal diameter of $3 \mathrm{~mm}$ has been inserted on a metallic cylinder with a diameter of $8 \mathrm{~mm}$, used as a support. Following the procedure already introduced in [22], a mechanical deformation has been applied along the braided sheath, until a bellows cylindrical structure appears. This manual process produces an effect that is strictly related to the mechanical design of the fibers of the sheath. By applying heat with a heat gun at $350^{\circ} \mathrm{C}$ for a few minutes, we obtain a thermal yielding. After the mechanical/thermal treatment, it has an outer diameter of $10 \mathrm{~mm}$ and a total length of $195 \mathrm{~mm}$. This configuration increases the elongation performances, by maintaining a radial containment effect of the internal elastic chamber. The above-mentioned McKibben-based actuator represents the starting point for development of our module that embeds three flexible fluidic actuators $120^{\circ}$ spaced and three cables that are $60^{\circ}$ spaced respect to the previous elements (Fig. 2). 


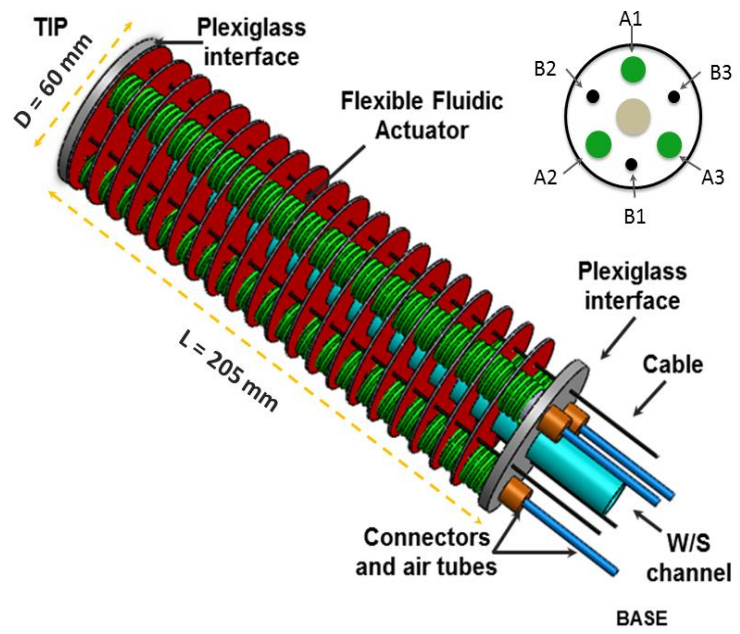

Figure 2. CAD model of the single module. In the upper section on the right there is a module cross section with three chambers (A1, $\mathrm{A} 2$ and $\mathrm{A3})$ and three cables (B1, B2 and B3).

All the components are concentric respect to an internal channel (a commercial tube used as a shower hose) that provides water and/or soap. The single module is $60 \mathrm{~mm}$ in diameter and $205 \mathrm{~mm}$ in length, as shown in Fig. 2, and the total weight is $180 \mathrm{~g}$. All the cables and chambers are decoupled, each one has a dedicated activation line for respectively tension and pressure regulation. One of the main issues we faced during the preliminary tests was represented by the lateral buckling of the system. The soft fluidic actuators can be visualized as elongating elements with no transversal constraints thus they experience spurious bending; they do not possess a preferential bending direction, but their deformation is guided by the external applied forces and constraints. In order to avoid this phenomenon and steer the actuators' force in the right directions, a flexible helicoidal structure has been inserted along the entire module thus constraining the lateral movement of the actuators. It is custom made and hosts the three flexible fluidic actuators, the three cables and the internal channel. The main advantage in using such a stabilizing structure is the possibility to guide the application force of the flexible fluidic actuators without affecting neither motion performances nor the overall passive flexibility of the system. The manufacturing process is multi-phase due to the number of elements the module is made of. We started by connecting the three flexible fluidic actuators between two thin plexiglass bases (thickness $=2$ $\mathrm{mm}$ ); then we have inserted the helicoidal structure and fixed it to the extremities of the module. The last part concerns the insertion of the three cables and the internal channel for the water/soap. As last step, the external sheath of the actuators have been anchored to the helicoidal structure thus avoiding sliding effects during operation.

\section{Experimental set-up}

The module is mounted on an acrylic box and inserted into the experimental set-up (Fig.3) consisting of the pneumatic circuit for controlling the pressure in each valve independently. Three proportional pressure micro regulator

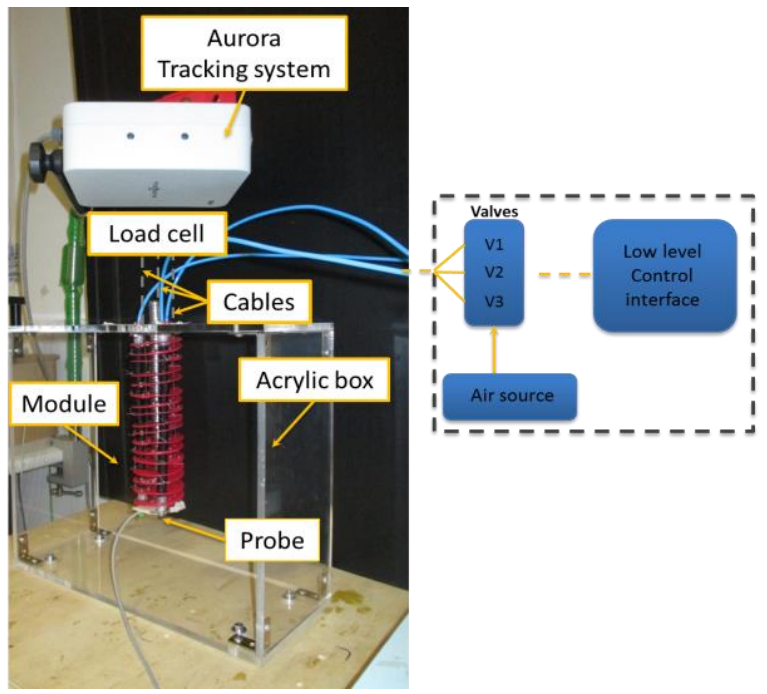

Figure 3. Experimental set-up

valves (Camozzi, K8P-0-E522-0) were used to modulate the air pressure inflated in each flexible fluidic actuator from 0 bar to 1.2 bar. A compressor (Junior 30 Werther International S.p.A.) was connected to the circuit as air source. A dedicated custom ROS interface allows to set the working pressure. Cable tensioning has been produced by connecting them to a load cell moving on a rail. For tracking the position of the single module in the space, an Aurora ${ }^{\circledR}$ Tracking system (Northern Digital Inc.) has been used with a six-DOF probe fixed on the tip of the module.

\section{Preliminary experimental evaluation}

The single module has been characterized through experimental tests aiming at evaluating its motion capabilities in the 3D space. In order to have a complete and quantitative description we measured elongation, shortening and bending capabilities. In particular the elongation and shortening evaluations have been carried out by pressurizing all the three chambers or by tensioning all the three cables respectively. On the other side, for the bending evaluation, we performed two different patterns of activation. They have the purpose of quantifying the force required by the cables for performing a $180^{\circ}$ rotation of the module tip after the maximum pressurization capability of the fluidic chambers.

Pattern 1: Activation of a single chamber (A1) from a pressure of 0 bar to $1.2 \mathrm{bar}$ (safety limit before damaging the actuators), without cable activation. When the maximum pneumatic pressure is reached, the activation of the opposite cable (B1) starts until a $180^{\circ}$ bending angle is achieved.

Pattern 2: Activation of two chambers (A2 and A3) in the same range of pressure as before without tensioning the cables. Again, when the maximum pneumatic pressure is reached, the co-activation of the two opposite cables (B2 and B3) is introduced. The maximum applied tension comes out from a $180^{\circ}$ rotation of the module.

The last part of the experimental characterization aims at defining the boundaries of the workspace for the different patterns of activation. We recorded the positions generated by a maximum pressurization ( $\operatorname{Pmax}=1.2$ bar) of all the single chambers (A1, A2 and A3) and the combination of 
them (A1-A2, A1-A3, A2-A3). Moreover, intermediate positions between the previous ones have been acquired by fixing one chamber at Pmax and setting the other one at $75 \%$ of the maximum pressure (corresponding to 0.85 bar). Regarding the tension in the cable, it has been applied at different steps. In pattern 1 (one chamber and one cable), a tension of $0.5 \mathrm{~N}, 1 \mathrm{~N}, 1.5 \mathrm{~N}$ and $2 \mathrm{~N}$ has been used, while in pattern 2 (with the double chambers), a tension of $0.3 \mathrm{~N}$, $0.5 \mathrm{~N}, 0.7 \mathrm{~N}$ and $1 \mathrm{~N}$ has been applied. Each test has been repeated three times, with the same identical conditions. The final result comes out from the mean value among the tests. The position and orientation of the module tip were acquired with the Aurora ${ }^{\circledR}$ Tracking system for 3 seconds, for each pressure value. In addition, images have been acquired at each step for each test, in order to measure the bending angles of the tip respect to the fixed base of the module.

\section{RESULTS AND DISCUSSION}

The single module has been studied and characterized in order to evaluate its performances in terms of shortening, elongation and bending capabilities. The flexible fluidic actuators are combined with cables, according to a specific pattern of activation. The idea of starting by activating the three chambers respect to the cables has been decided in order to firstly exploit the global movement of the module, due to the fluidic capabilities and then to adjust the position (curvature and orientation) with the cables tensioning. The following section summarizes all the results, starting from the characterization of the single McKibben-based actuator at increasing pressure values.

\section{A. Flexible fluidic actuator characterization}

Before embedding the actuator in the module, it has been tested in order to assess its elongation capability respect to the pressure. This preliminary test demonstrates how the bellows-type design of the external sheath enables an elongation up to $85 \%$ respect to its initial length (Fig. 4). Another consideration regards the pressure threshold before starting the motion. The system does not respond until the pressure reaches 0.2 bar. It means that this pressure is only sufficient to deform the walls of the internal balloon and after this threshold the pressure starts to work against the braided structures and generates elongation.

\section{B. Shortening}

The data describing the shortening capabilities of the module have been acquired by a simultaneous tensioning of the three cables. Starting from a total length of $205 \mathrm{~mm}$, the module achieves a shortened position of $155 \mathrm{~mm}$ by applying $18 \mathrm{~N}$. There is a $25 \%$ reduction of the length. This result is apparently not involved into the design requirements but, it could be useful in all the tasks where the tip has to reach a target position near the base of the system mounted on the wall. It implies that a multi-bending of the module is required and synergized with a shortening movement.

\section{Elongation}

Elongation performances have been tested through the acquisition of the maximum length achieved by activating

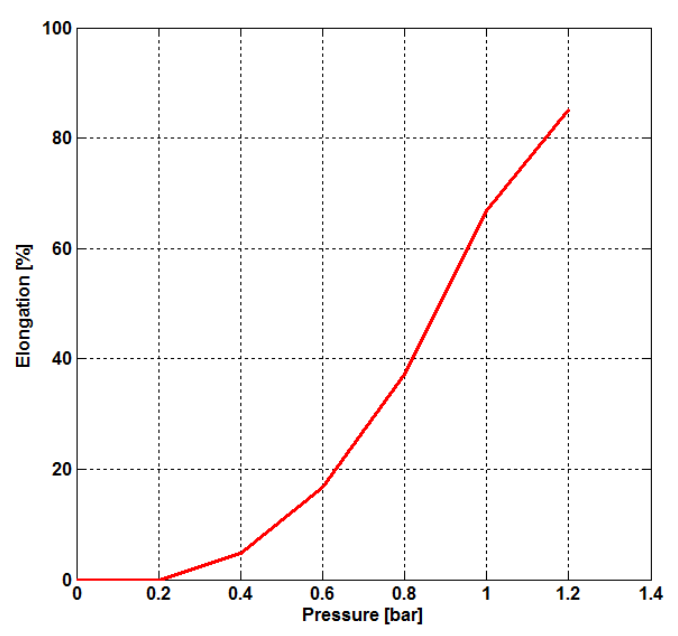

Figure 4. Pressure [bar] - Elongation [\%] relationship for the flexible fluidic McKibben-based actuator.

all the fluidic chambers with Pmax and leaving the three cables free. This configuration allows module to expand until a final length of $345 \mathrm{~mm}$, by increasing its length of $68 \%$.It means that each chamber, starting from an initial length of $195 \mathrm{~mm}$, as presented in the previous section, undergoes to $72 \%$ of deformation respect to its initial length. The lower elongation achieved by the flexible fluidic actuators when embedded into the module, respect to the performances of the free actuator (reported in section III-A), is caused by the constraints and limitations imposed by the structural elements of the module (e.g. the helicoidal structure). However, the final elongation covered by the module agrees with the expected outcomes. Considering that our arm will be made of a proximal module of $200 \mathrm{~mm}$ and two identical modules capable of an elongation up to 345 $\mathrm{mm}$ (all the three modules are interconnected with two interfaces $25 \mathrm{~mm}$ in thickness) the maximum length that could be obtained will be around $900 \mathrm{~mm}$. This result is encouraging because it guarantees to cover the worst case where the arm has to achieve a point that is $750 \mathrm{~mm}$ far from the wall.

\section{Bending}

Bending performances (shown in Fig. 5) have been analysed for increasing pressure values. In particular, even if the working pressure range was from 0 bar to 1.2 bar (with 0.1 bar of resolution), we noticed no movements from 0 bar to 0.5 bar, due to the non-linear response of the actuator, as mentioned in the section III-A. This gap $(0.5$ bar respect to 0.2 bar), quite different from the previous threshold, is probably due to the further constraints introduced by the module manufacturing (respect to the single free element).

Pattern 1: Fig. 6 represents the bending angle $\left(118^{\circ} @\right.$ Pmax) of the tip due to the activation of the single chamber (A1). The overall movement of the module in the 3-D space, concerning the pattern 1 is shown in Fig. 5A. In this experiment, the tip undergoes a displacement along the $\mathrm{z}-$ axis of approximately $110 \mathrm{~mm}$ respect to the rest position, against gravity effects. This value can be extrapolated from the xz-view of the system in Fig. 5B. 


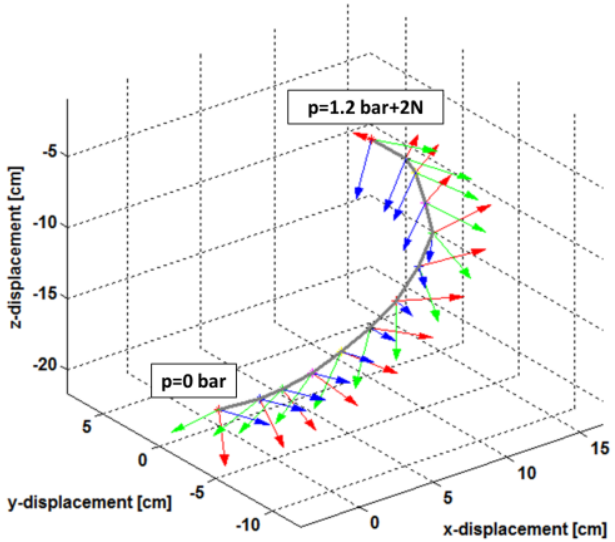

A)

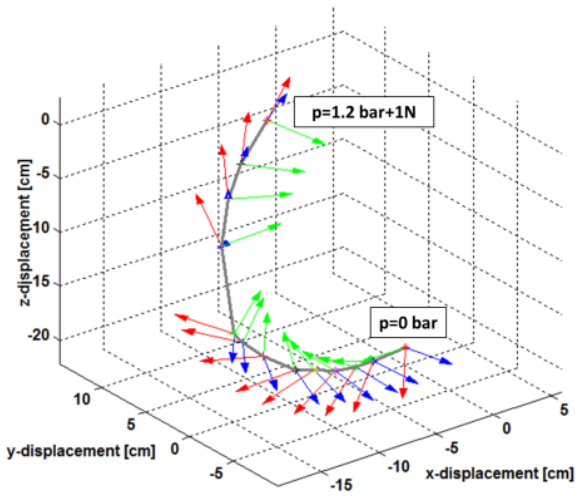

D)

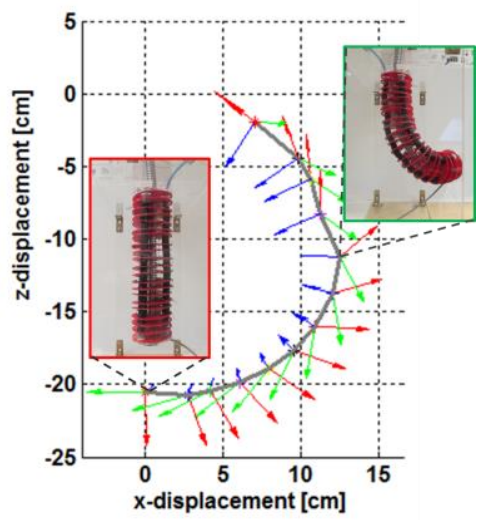

B)

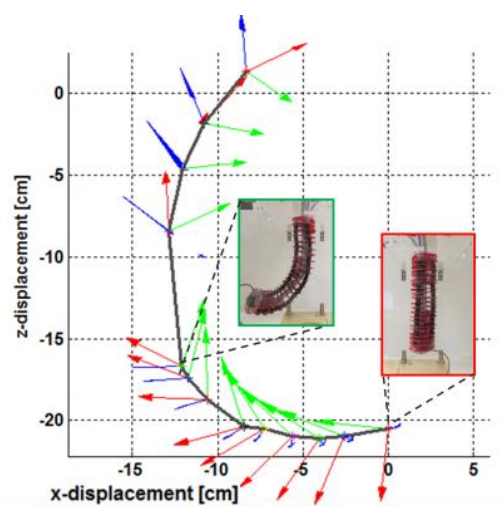

E)

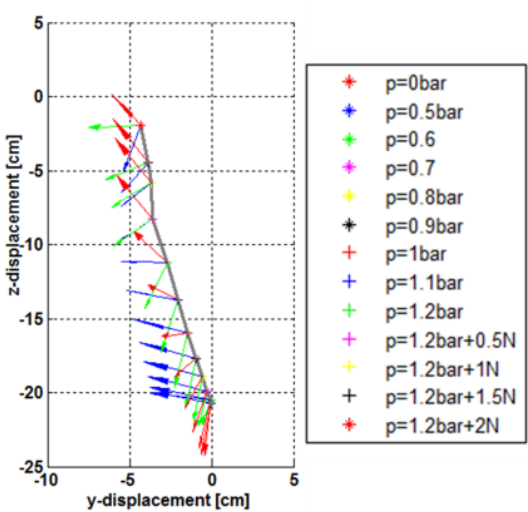

C)

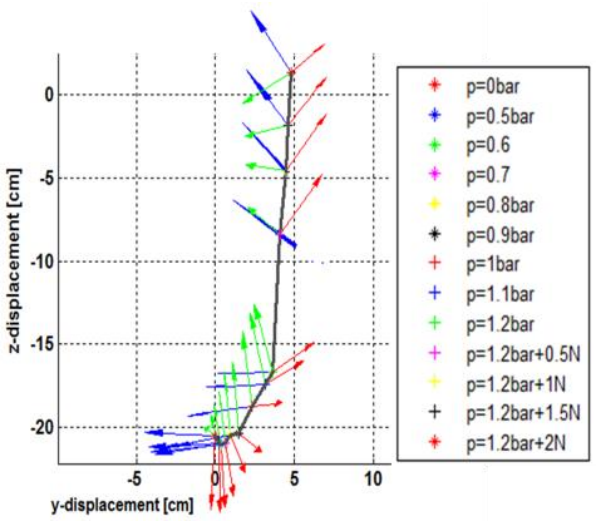

F)

Figure 5. Bending performances of the two activation patterns. A) 3D view of pattern $1(A 1+B 1)$; B) xz view of pattern 1;C) yz view of pattern 1; D) 3D view of pattern 2 (A2-A3 + B2-B3); E) xz view of pattern 2; F) yz view of pattern 2. Note: These diagrams present the displacements axes in $\mathrm{cm}$, this choice is just for a better visualization of the results. All the data presented in the written test are in mm. (Also for Fig. 7)

Another observation regards the role played by the single opposite cable (B1) respect to the overall movement of the module. A $180^{\circ}$ rotation of the module is achieved by applying $2 \mathrm{~N}$ of tension in $\mathrm{B} 1$. The behavior of the module respect to the yz plane (Fig. 5C) shows a lateral displacement of 25 mm (@ Pmax), meaning that the module is not moving on the plane. This behavior is probably due to a slight asymmetry introduced during the manual manufacturing procedure of the module (not easily identifiable when the module is at rest). By forcing a straight position of the chambers respect to the plexiglass bases, this effect can be removed.

Pattern 2: As shown in Fig. 6, the maximum bending angle is close to the previous case but, the displacement of the tip is less pronounced. Respect to the previous pattern of activation, by co-activating two chambers (A2-A3) it is possible to observe a different response of the module, as shown in Fig 5D. More in detail, Fig. 5E shows that by applying Pmax to both the chambers, the module tip undergoes a vertical displacement of $30 \mathrm{~mm}$. As consequence, the module is firstly elongated and then, the distal portion of the total length produces the bending. Also in this case it is possible to observe a lateral displacement of approximately30 mm (@ Pmax), due to the asymmetry of the module (Fig. 5F). Regarding the effect produced by the cables co-activation (B2-B3), by applying $1 \mathrm{~N}$ in each cable, the module is able to change its position in the space, by

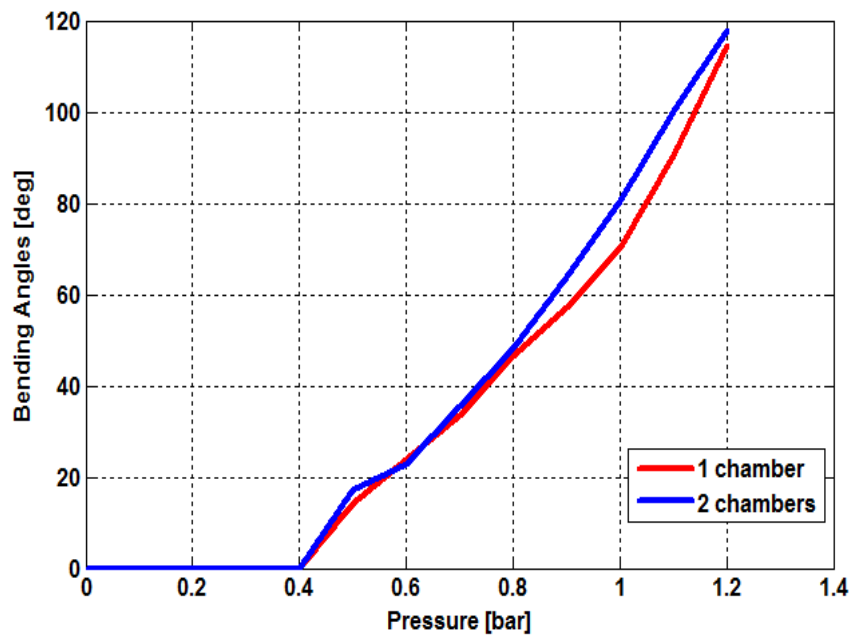

Figure 6. Bending angle at increasing pressure values. One flexible fluidic actuator (red line), two flexible fluidic actuators (blue-line). 


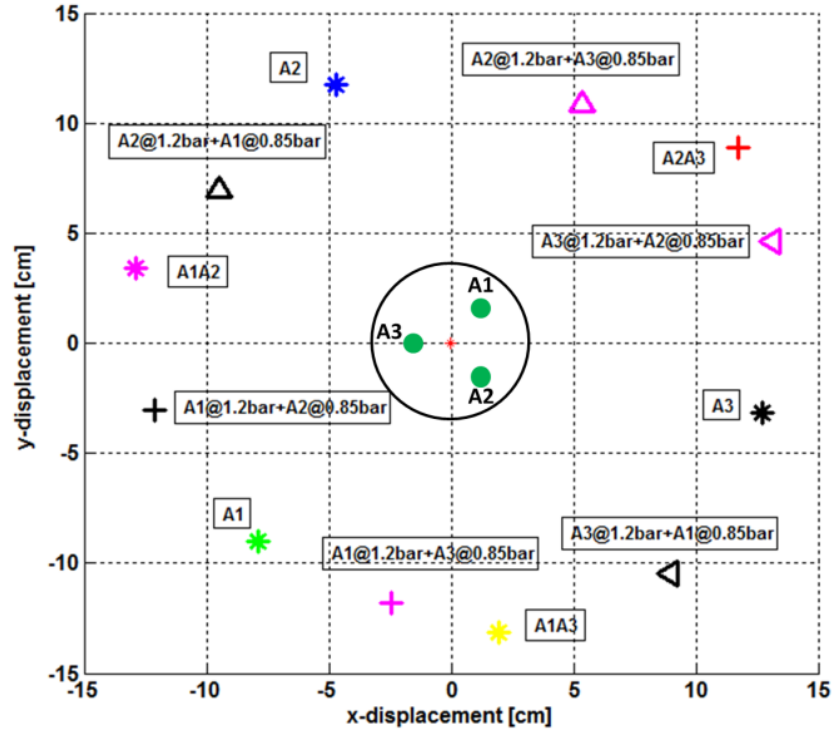

Figure 7. Module workspace (xy view): maximum positions reached by the tip for different patterns of activation.

producing $180^{\circ}$ rotation respect to the base. Surprisingly, in order to achieve the same tip bending angle, the two activation patterns require the same amount of pulling force on the cables $(2 \mathrm{~N}$ versus $2 \times 1 \mathrm{~N})$ but different chamber pressure (1.2 bar versus $2 \times 1.2$ bar), which may also justify the bigger elongation achieved in the pattern 2 .

\section{E. Workspace}

The last measurement aims at defining the boundaries of the reachable positions of the module tip. Fig. 7 represents the module performances in the xy-plane (top view). In the 3Dspace all these points are located at different z-positions respect to the starting position. In addition to the detailed tracking of the module tip pointed out with the pattern 1 and 2 , this last elaboration allows the complete definition of the positions described by the tip of the module.

\section{CONCLUSIONS}

The study proposed in this work faces, for the first time, the challenge of developing a robotic modular manipulator that safely interacts with the user for accomplishing bathing tasks, one of the most critical ADLs. The concept moves from the outcomes achieved in the field of soft robotics, that provides suitable technologies in order to address all the requirements for the target application. The main achievements of the proposed work regard the design, development and testing of a soft module based on three flexible fluidic actuators in combination with three cables, as part of a modular soft robotic shower arm. The experimental part is focused on the characterization of the a single module performances in relation to the final purpose of the system. These preliminary results, promising and encouraging, represent the starting point for the development of the first soft assistive robot for personal care of elderly people.

\section{REFERENCES}

[1] S. W. Brose, D. J. Weber, B. A. Salatin, G. G. Grindle, H. Wang, J. J. Vazquez, and R. A. Cooper, 'The role of assistive robotics in the lives of persons with disability', Am J Phys Med Rehabil, vol. 89, no. 6, pp. 509-521, Jun. 2010.

[2] F. G. Miskelly, 'Assistive technology in elderly care', Age Ageing, vol. 30 , no. 6 , pp. 455-458, Jan. 2001.

[3] http://ec.europa.eu/eurostat/statistics-explained/index.php/

[4] S. Katz, 'Studies of illness in the aged, The index of ADL: A standardized measure of biological and psychosocial function', Jama, vol. 12, p. 919, 1963.

[5] C. B. Fausset, A. J. Kelly, W. A. Rogers, and A. D. Fisk, 'Challenges to Aging in Place: Understanding Home Maintenance Difficulties', J Hous Elderly, vol. 25, no. 2, pp. 125-141, 2011.

[6] D. D. Dunlop, S. L. Hughes, and L. M. Manheim, 'Disability in activities of daily living: patterns of change and a hierarchy of disability.', Am J Public Health, vol. 87, no. 3, pp. 378-383, Mar. 1997.

[7] C.A. Smarr, C. B. Fausset, and W. A. Rogers, 'Understanding the Potential for Robot Assistance for Older Adults in the Home Environment', 2011.

[8] T. Bock, C. Georgoulas, and T. Linner, 'Towards robotic assisted hygienic services: Concept for assisting and automating daily activities in the bathroom', Gerontechnology, vol. 11, no. 2, p. 362, Jun. 2012.

[9] http://www.seatedshower.com/

[10] http://www.drivemedical.co.uk/sections/bathroom-toilet-aids

[11] E. A. Kirchner, J. C. Albiez, A. Seeland, M. Jordan, and F. Kirchner, F. "Towards Assistive Robotics for Home Rehabilitation". In Biodevices, 2013, pp. 168-177.

[12] Q. Meng and M. H. Lee, 'Design issues for assistive robotics for the elderly', Advanced Engineering Informatics, vol. 20, no. 2, pp. 171186, Apr. 2006.

[13] A. Loeve, P. Breedveld, and J. Dankelman, 'Scopes Too Flexible... and Too Stiff', IEEE Pulse, vol. 1, no. 3, pp. 26-41, Nov. 2010.

[14] G. Immega and K. Antonelli, 'The KSI tentacle manipulator', in IEEE International Conference on Robotics and Automation, 1995. Proceedings, 1995, vol. 3, pp. 3149-3154 vol.3.

[15] W. McMahan, V. Chitrakaran, M. Csencsits, D. Dawson, I. D. Walker, B. A. Jones, M. Pritts, D. Dienno, M. Grissom, and C. D. Rahn, 'Field trials and testing of the OctArm continuum manipulator', in 2006 IEEE International Conference on Robotics and Automation, 2006. Proceedings ICRA 2006, ,pp. 2336-2341.

[16] W. McMahan, B. A. Jones, and I. D. Walker, 'Design and implementation of a multi-section continuum robot: Air-Octor', in 2005 IEEE/RSJ International Conference on Intelligent Robots and Systems, 2005. (IROS 2005), 2005, pp. 2578-2585.

[17] M. B. Pritts and C. D. Rahn, 'Design of an artificial muscle continuum robot', in 2004 IEEE International Conference on Robotics and Automation, 2004. Proceedings. ICRA '04, 2004, vol. 5, pp. 4742-4746 Vol.5.

[18] A. De Greef, P. Lambert, and A. Delchambre, 'Towards flexible medical instruments: Review of flexible fluidic actuators', Precision Engineering, vol. 33, no. 4, pp. 311-321, Oct. 2009.

[19] Q. Yang, L. Zhang, G. Bao, S. Xu, and J. Ruan, 'Research on novel flexible pneumatic actuator FPA', in 2004 IEEE Conference on Robotics, Automation and Mechatronics, 2004, vol. 1, pp. 385-389 vol.1.

[20] M. De Volder, A. J. M. Moers, and D. Reynaerts, 'Fabrication and control of miniature McKibben actuators', Sensors and Actuators A: Physical, vol. 166, no. 1, pp. 111-116, Mar. 2011.

[21] R. Kang, D. T. Branson, T. Zheng, E. Guglielmino, and D. G. Caldwell, 'Design, modeling and control of a pneumatically actuated manipulator inspired by biological continuum structures', Bioinspir. Biomim., vol. 8, no. 3, p. 036008, 2013.

[22] T. Ranzani, M. Cianchetti, G. Gerboni, I. D. Falco, and A. Menciassi, 'A Soft Modular Manipulator for Minimally Invasive Surgery: Design and Characterization of a Single Module', IEEE Transactions on Robotics, vol. 32, no. 1, pp. 187-200, Feb. 2016. 